\title{
Osteoartritis (artrosis) de la articulación temporomandibular
}

\section{Temporomandibular joint osteoarthritis (arthrosis)}

\author{
Marco Sánchez T. ${ }^{1}$, Walter Becerra B. ${ }^{2}$
}

'Universidad Privada de Tacna, Facultad de Ciencias de la

Salud, Escuela Profesional de

Odontología. Tacna, Perú.

${ }^{2}$ Hospital de Emergencias Villa

el Salvador. Lima, Perú

Los autores declaran no tener conflicto de interés.

Recibido el 4 de marzo de 2020. Aceptado el 17 de junio de 2020 .

Correspondencia: Marco Sánchez T. Av. Jorge Basadre Grohmann $\mathrm{s} / \mathrm{n}$, Pocollay Universidad Privada de Tacna. Tacna, Perú. Email: marcosanchez2183@ gmail.com

\section{Resumen}

La osteoartritis de la articulación temporomandibular (ATM) es un desorden degenerativo de etiología multifactorial, que requiere un manejo interdisciplinario. Se presenta entre el $8 \%$ al 16\% de la población y está asociada al exceso de carga articular, trauma, factores genéticos, parafunciones y desarreglos internos de la articulación, también se reconoce una alta frecuencia de otalgia refleja y tinnitus en pacientes con desorden degenerativo de la ATM. El uso combinado de tomografía computarizada y resonancia magnética permite observar las variaciones morfológicas en los tejidos duros y blandos de la estructura articular. El tratamiento no invasivo incluye la indicación de férulas de reposicionamiento mandibular, además del uso de antiinflamatorios y preferencia por inhibidores selectivos de la ciclooxigenasa-2 (COX-2). La inyección intraarticular de corticoides disminuye a largo plazo la inflamación de los tejidos articulares y mejora la movilidad, mientras que el ácido hialurónico parece disminuir la fricción en los espacios articulares. Los procedimientos quirúrgicos como artroscopia, condilectomía, artroplastia, reemplazo autógeno de disco o reemplazo total de la articulación deben ser reservados para los casos en donde la intervención no invasiva no muestra mejoría clínica.

Palabras clave: osteoartritis, articulación temporomandibular, epidemiología, etiología, tratamiento.

\begin{abstract}
Osteoarthritis of the temporomandibular joint (TMJ) is a degenerative disorder of multifactorial etiology, which requires interdisciplinary management. It occurs between $8 \%$ to $16 \%$ of the population and is associated with excess joint load, trauma, genetic factors, parafunctions and internal disorders of the joint, a high frequency of reflex otalgia and tinnitus is also recognized in patients with degenerative TMJ disorder. The combined use of computed tomography and magnetic resonance allows to observe the morphological variations in the hard and soft tissues of the joint structure. Non-invasive treatment includes the indication of mandibular repositioning splints, in addition to the use of anti-inflammatory drugs and preference for selective cyclooxygenase-2 inhibitors (COX-2). Intra-articular injection of corticosteroids reduces inflammation of the articular tissues and improves mobility in the long term, while hyaluronic acid seems to decrease friction in the articular spaces. Surgical procedures such as arthroscopy, condylectomy, arthroplasty, autogenous disc replacement, or total joint replacement should be reserved for cases where non-invasive intervention shows no clinical improvement.
\end{abstract}

Keywords: osteoarthritis, temporomandibular joint, epidemiology, etiology, treatment.

\section{Introducción}

La articulación temporomandibular (ATM) está relacionada con funciones complejas como la masticación, deglución y fonética. Durante las funciones del sistema estomatogná- tico pueden ocurrir alteraciones o desarreglos estructurales; sin embargo, la tolerancia fisiológica permite que estos eventos sucedan sin que exista un efecto adverso sobre las estructuras que componen el sistema ${ }^{1}$. Cuando las alteraciones sobrepasan la tolerancia fisiológica 
ocurrirá un cambio que puede comprometer a la ATM, músculos y dientes ${ }^{1,2}$. Las superficies articulares están revestidas por fibrocartílago que permite soportar altos niveles de estrés y sobrecarga articular y, por otro lado, es capaz de repararse y remodelarse producto de estímulos nocivos ${ }^{1,3}$.

Los desórdenes temporomandibulares (DTM) pueden clasificarse en: desórdenes de los músculos de la masticación, desórdenes de la ATM, hipomovilidad mandibular crónica y desórdenes del crecimiento ${ }^{1}$. Los desórdenes de la ATM incluyen a los desórdenes de carácter inflamatorio y se denominan de acuerdo a la zona afectada, pudiendo ser: capsulitis, sinovitis, retrodiscitis y osteoartritis ${ }^{1,3}$. La artrosis u osteoartritis (OA) es una enfermedad crónico-degenerativa que se caracteriza por la destrucción gradual y progresiva del cartílago que recubre la superficie articular particularmente en rodillas, caderas, hombros, manos, tobillos y columna vertebral ${ }^{4,5}$. También puede presentarse en la ATM, y son muchos los factores que han sido señalados como responsables del desarrollo de la OA en la ATM, entre ellos; sobrecarga articular, bruxismo, masticación unilateral, factores genéticos, alteraciones hormonales y desarreglos internos de la articulación ${ }^{6,7}$. Además, la OA se produce por un desbalance en los procesos metabólicos mediados por los condrocitos y se caracteriza por una degradación gradual de los componentes de la matriz extracelular del fibrocartílago con o sin factores inflamatorios secundarios ${ }^{8,9}$.

La OA de la ATM representa una condición inflamatoria de bajo nivel que ocasiona una erosión del cartílago articular y degradación del hueso subcortical adyacente ${ }^{10,11}$. Cuando las alteraciones óseas están activas la condición se denomina osteoartritis; a medida que ocurre la remodelación, la condición se torna estable aún cuando la morfología ósea permanezca alterada, y se le denomina osteoartrosis ${ }^{1}$. La osteoartrosis es considerada una adaptación mal sucedida de la ATM cuando es sometida a fuerzas mecánicas como las alteraciones del complejo cóndilo-disco ${ }^{12}$. Como su etiología, fisiopatología y tratamiento son los mismos, los términos osteoartritis y osteoartrosis son empleados como sinónimos, pudiendo referirse a ellas como enfermedad articular degenerativa ${ }^{11}$. También se reconoce una alta frecuencia de otalgia refleja y tinnitus en pacientes con $\mathrm{DTM}^{1,2}$, por lo que es necesario analizar el comportamiento degenerativo de la ATM en este grupo de pacientes.

El presente artículo tiene por objetivo revisar los aspectos de epidemiología, etiología, fisiopatología, diagnóstico y tratamiento relacionados con la OA de la ATM, desde una perspectiva interdisciplinaria considerando que es una condición bastante frecuente en los pacientes que acuden a la consulta médica y odontológica y que continúa siendo un apartado discutido entre los especialistas, esta particularidad supone un entendimiento claro y completo por parte de los profesionales.

\section{Epidemiología}

Dentro de los desórdenes de la ATM que incluyen los trastornos del complejo cóndilo-disco, hipomovilidad, enfermedad articular degenerativa y trastornos congénitos o del desarrollo ${ }^{2}$, la OA es la afección más frecuente y se presenta entre el $8 \%$ al $16 \%$ de la población general y puede tener un compromiso uni o bilateral ${ }^{13}$. Bernhardt y cols. encontraron una prevalencia de OA de la ATM a través de examen clínico y resonancia magnética del 25\% en un grupo de pacientes con edades de 20 a 49 años ${ }^{14}$. Por otro lado, Schimitter y cols. encontraron una prevalencia del $70 \%$ en un grupo de personas de 73 a 75 años. Un dato interesante es que la OA de la ATM es dolorosa solo en estadios tempranos y se vuelve no dolorosa con el paso del tiempo ${ }^{15}$, además, se reconoce que los signos radiográficos de la OA se incrementan mientras el dolor disminuye ${ }^{16}$. Torrealba y cols. encontraron que la prevalencia de signos radiográficos de $\mathrm{OA}$ en una población chilena fue del 35\%, siendo más frecuente en pacientes mayores de 50 años ${ }^{17}$. Los estudios refieren también que existe una alta preferencia de presentar OA de la ATM en mujeres, esto puede ser debido al polimorfismo del gen receptor de estrógenos alfa y puede estar asociado con el incremento de susceptibilidad al dolor en pacientes mujeres con OA de la $\mathrm{ATM}^{8,18,19}$. $\mathrm{Al}$ respecto, Cömert y cols. reportaron una frecuencia de OA de la ATM del 85,5\% en pacientes mujeres frente a un $14,5 \%$ en pacientes hombres ${ }^{20}$; datos similares han sido 
reportados en otros estudios ${ }^{9,21,22}$. Después de la OA de la ATM, la artritis reumatoide, artritis psoriásica y espondilitis anquilosante son las enfermedades reumatológicas que presentan mayor ocurrencia de alteración degenerativa a nivel de la $\mathrm{ATM}^{23}$. La ocurrencia de OA de la ATM ha sido reportada entre el $40 \%$ y $96 \%$ de pacientes con artritis idiopática juvenil, afectando de manera importante la función en este grupo de pacientes ${ }^{24}$. Goupille y cols. identificaron que el $69 \%$ de pacientes con artritis reumatoide desarrolló lesiones erosivas císticas en la $\mathrm{ATM}^{25}$, además, se sabe que se sigue el mismo patrón destructivo que en otras articulaciones ${ }^{26}$. Por otro lado, en la literatura se reconoce una alta frecuencia de otalgia refleja y tinnitus en pacientes con $\mathrm{DTM}^{1,27,28}$, al respecto Macedo y cols. encontraron una asociación significativa entre la presencia de DTM y otalgia (odds ratio $=2,3$ ) en pacientes adultos mayores ${ }^{29}$. Molina y cols. indican que los pacientes con dolor en las inserciones del disco articular y OA de la ATM presentaron mayor frecuencia de otalgia $(48,6 \%$ y $77,5 \%$ respectivamente), al compararlos con un grupo control $(19,4 \%)^{30}$.

\section{Etiología}

Se han identificado como factores de riesgo para la ocurrencia de OA de la ATM el exceso de carga articular, trauma, factores genéticos, parafunciones (bruxismo, masticación unilateral, hábitos de mordedura de objetos), oclusión inestable y desarreglos internos de la articulación como el desplazamiento anterior del disco $7,10,11,31,32$, estos factores pueden ocurrir aislados o pueden estar interrelacionados y/o coexistir. Sin embargo, no todos los pacientes con estas características desarrollarán OA de la ATM, esto dependerá de la capacidad adaptativa de cada paciente asociada a otros factores como la edad, enfermedades sistémicas y alteraciones hormonales ${ }^{33}$.

Cuando la carga funcional de la ATM aumenta, se produce una adaptación funcional por medio de la remodelación de la superficie articular ${ }^{10,34}$. Sobrepasada la capacidad adaptativa, ocurrirá una serie de eventos como la liberación de radicales libres, citoquinas, catabolitos y enzimas que degradan la matriz extracelular, promoviendo la degeneración de las superficies articulares ${ }^{35}$. Tanto la frecuencia como la severidad de la OA de la ATM parecen incrementarse con la edad, se sabe que a los 40 años el $20 \%$ de la población presenta signos clínicos de $\mathrm{OA}^{36}$, mientras que entre los 73-75 años el $70 \%$ ha desarrollado signos radiográficos evidentes de la enfermedad a nivel de la $\mathrm{ATM}^{15}$; Takano y cols. han reportado que este hecho puede deberse a los incrementos en los contenidos minerales en el disco articular provocando un incremento en la fragilidad del mismo, reduciendo así su capacidad a soportar las sobrecargas ${ }^{37}$. Holmes y cols., citado por Tanaka y cols. señalan que la disminución progresiva del peso molecular del ácido hialurónico contenido en el cartílago articular está relacionado con el incremento de la edad y esto afectaría las propiedades mecánicas y del comportamiento de fluidos en el cartílago, incrementando las posibilidades de presentar procesos degenerativos ${ }^{11}$.

Las enfermedades sistémicas pueden alterar el metabolismo del fibrocartílago afectando su respuesta adaptativa frente a las sobrecargas de la articulación; desórdenes autoinmunes, endocrinos, nutricionales, enfermedades metabólicas y procesos infecciosos podrían estar relacionados con este evento ${ }^{10}$. La diferencia hormonal entre hombres y mujeres puede exacerbar la degeneración del cartílago y hueso articular en la $\mathrm{ATM}^{8,38}$. Arnett y Gunson señalan que la predisposición femenina a la remodelación disfuncional de la ATM podría estar en parte atribuida a la modulación de las respuestas biológicas de los tejidos articulares a las hormonas sexuales, particularmente con los niveles bajos de estrógenos y prolactina ${ }^{33}$. Kang y cols. sugieren que el polimorfismo del gen receptor de estrógenos alfa específicamente el haplotipo Px está asociado con la susceptibilidad al dolor en pacientes con OA de la $\mathrm{ATM}^{19}$. Guarda-Nardini y cols. identificaron que los desórdenes de la ATM, incluidos aquellos relacionados con artritis/artrosis, son más frecuentes en pacientes con un rango de edad de 51,9 $\pm 14,5$ años y que suelen estar acompañados de sonidos crepitantes, aunque el dolor crónico no está relacionado con la gravedad de los cambios a nivel óseo ${ }^{39}$. Los cambios óseos degenerativos de la ATM son muy prevalentes en pacientes adultos (quiste 
subcondral: 63,3\%; osteofitos: $60 \%$; osteoporosis/osteopenia: $38,5 \%$ ) y las mujeres suelen ser las más afectadas ${ }^{40}$.

\section{Fisiopatología}

La OA de la ATM comparte la fisiopatología de articulaciones sinoviales más grandes como las rodillas. Cargas mecánicas excesivas y daño del cartílago articular son descritos como los que desencadenarían el desbalance de la homeostasis de la matriz. La maloclusión, asimetría mandibular y sobrecarga muscular han sido considerados como posibles causas para el desarrollo de artrosis de esta articulación ${ }^{41,42}$. El condrocito mantiene la homeostasis de la matriz extracelular del cartílago. Tienen capacidad de liberar mediadores de la inflamación entre proteasas y citoquinas, factores de crecimiento, radicales libres y prostaglandinas. Fuerzas cíclicas o estáticas constantes aumentan el catabolismo de la matriz conllevando a la reorganización de fibras de colágeno y proteoglicanos de manera desordenada y como compensación se sintetiza más matriz e hipertrofia del tejido en zonas que reciben mayor presión y es donde se produce mayor cantidad de factores de crecimiento. El factor de crecimiento insulínico tipo I (IFG-I) es anabólico, tiene función disminuida en la osteoartritis pese a que su síntesis está incrementada. El óxido nítrico (NO), presente normalmente en el cartílago articular, inhibe la respuesta del condrocito a este factor de crecimiento debido a que está presente en mayor cantidad en el cartílago con osteoartritis. El factor de crecimiento transformante beta 1 (TGF-1) en ratones transgénicos ha demostrado tener un papel en el inicio de la disminución de la densidad mineral ósea y mayor recambio del hueso subcondral. Se observó también en este modelo que en los condrocitos condilares existe regulación positiva de metaloproteinasas de la matriz (MMP-9 y MMP-13) así como factor de crecimiento vascular endotelial (VEGF) y densidad ósea variable ${ }^{43,44}$.

Las metaloproteinasas son enzimas proteolíticas controladas por los inhibidores de tejido de metaloproteinasas (TIMP). La MMP-3 está presente en niveles altos en líquido sinovial de pacientes con OA. Puede activar otras formas de MMP y ayudar a la progresión del daño en la matriz. También participan citoquinas como la interleuquina $1 \beta$ (IL-1 $\beta$ ) y el factor de necrosis tumoral alfa (TNF- $\alpha$ ), que facilitan la degradación del cartílago. La IL-1 $\beta$ está incrementada en el cartílago donde los condrocitos son estimulados a producir otras citoquinas al igual que en los sinoviocitos en la membrana sinovial, estimula proteasas e inhibe a los TIMP $^{44,45}$. Suprime la expresión de colágeno II, IX y la formación de proteoglicanos, así como la producción de $\mathrm{NO}$ y prostaglandinas. El TNF- $\alpha$ estimula a nivel sinovial la inflamación. La prostaglandina E2 (PGE2) está presente en altas concentraciones en el líquido sinovial de pacientes con OA de la ATM comparada con los de pacientes con OA de rodillas ${ }^{46}$.

Se describe una relación directa entre inflamación y estrés mecánico del cartílago. Los estímulos mecánicos en los condrocitos cultivados aumentan la producción de leucotrieno B4 (LTB4) y NO, así como MMP-9. La membrana sinovial participa liberando mediadores inflamatorios como IL- $1 \beta$ y TNF- $\alpha$ y en ella se observa proliferación de sinoviocitos en fases avanzadas de OA. La presencia de cristales de pirofosfato cálcico de hidroxiapatita mantiene el proceso inflamatorio a este nivel ${ }^{43,47-49}$. En el hueso subcondral se presenta desregulación de la osteoclastogénesis con aumento del ligando del receptor activador del factor nuclear kB (RANKL) y osteoprotegerina (OPG) conllevando a pérdida de hueso subcondral en OA de ATM. El dolor presente en la OA se podría explicar por la alta inervación a este nivel o por hiperexcitabilidad de terminaciones nerviosas aferentes. La disminución de mecanorreceptores podría participar en la reducción de la estabilidad articular facilitando el proceso degenerativo en la $\mathrm{OA}^{47}$. En la Figura 1 se resume el proceso fisiopatológico de la OA de la ATM.

\section{Diagnóstico}

\section{Diagnóstico clínico}

Los signos y síntomas clínicos de la OA están representados por ruidos articulares como el clicking y crepitación durante los movimientos mandibulares, limitación de los movimientos mandibulares y dolor asociado 


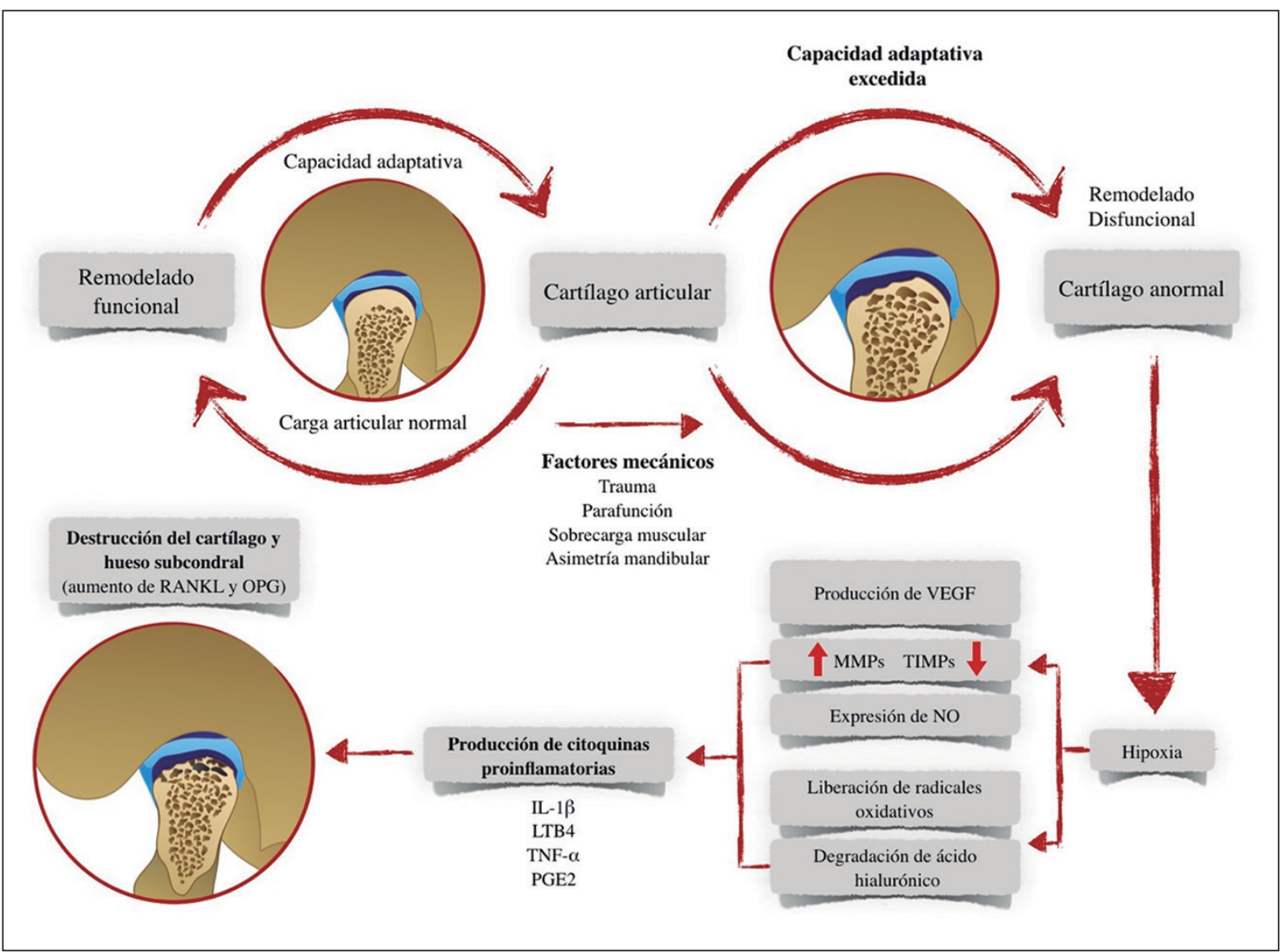

Figura 1. Esquema de la fisiopatología del proceso degenerativo de la osteoartritis de la articulación temporomandibular. VEGF: factor de crecimiento vascular endotelial, MMPs: metaloproteinasas, TIMPs: inhibidores de tejido de metaloproteinasas, NO: óxido nítrico, IL-1 $\beta$ : la interleuquina1 $\beta$, LTB4: leucotrieno B4, TNF- $\alpha$ : factor de necrosis tumoral alfa, PGE2: prostaglandina E2, RANKL: ligando del receptor activador del factor nuclear kB OPG: osteoprotegerina (adaptado y rediseñado de Tanaka y cols., $2008^{10}$ ).

a nivel articular y muscular ${ }^{1,9,32}$, en algunas circunstancias puede presentarse inmovilización de la articulación afectada, esto se explica por la formación de osteofitos marginales, que impiden los movimientos normales de la articulación $^{3}$. La OA suele ser dolorosa solo en los estadios iniciales, tornándose indolora a medida que la enfermedad progresa ${ }^{14}$. Martínez y cols. señalan que para diagnosticar a un paciente con OA de la ATM, este debe presentar crepitación a la auscultación de la ATM afectada, además de disminución en la movilidad de la ATM y desviación en la apertura hacia el lado afectado9. Cuando la OA de la ATM es bilateral puede ocasionar la presencia de una mordida abierta anterior, donde el paciente refiere una evolución progresiva, mostrando contactos oclusales solo a nivel del sector posterior ${ }^{2,33,34}$ (Figura 2).

\section{Diagnóstico por imágenes}

Los primeros estadios de OA de la ATM no generan cambios identificables por medio de la ayuda diagnóstica por imágenes. Los principales signos de OA de la ATM hallados por medio del diagnóstico por imágenes son: esclerosis del hueso subcondral, erosión, aplanamiento de las superficies articulares, deformación en la superficie condilar, osteofitos y disminución del espacio articular ${ }^{9,32,50}$. La radiografía panorámica, es la primera herramienta para identificar cambios estructurales a nivel de la ATM; sin embargo, la agudeza con la que puede evidenciar estos cambios debe ser evaluada con 


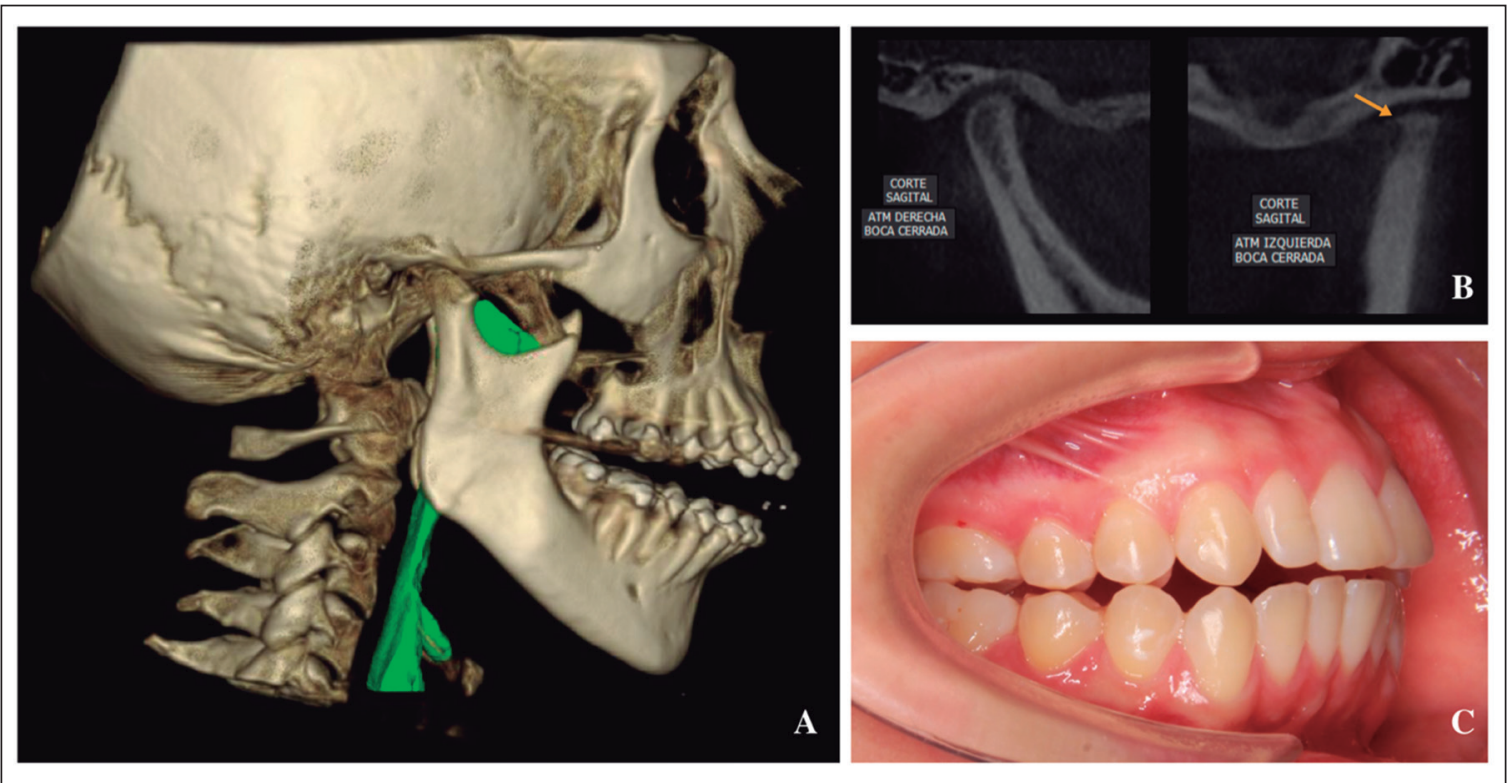

Figura 2. Paciente con osteoartritis bilateral de la articulación temporomandibular (ATM) y presencia de mordida abierta. (A) Reconstrucción 3D de imagen de tomografía computarizada de haz cónico. (B) Detalle de osteofito (flecha) en ATM izquierda. (C) Mordida abierta anterior.

cuidado, ya que en ella existe superposición de imágenes. Al ser una proyección infracraneal, el polo lateral del cóndilo queda superpuesto a la cabeza condílea, de tal manera que en la imagen el área que parece corresponder a la superficie subarticular superior del cóndilo en realidad es la superficie subarticular del polo medial ${ }^{1}$.

La tomografía computarizada (TC) puede ser empleada para detectar alteraciones de la ATM, sin requerir medios de contraste y permite reconstrucciones en los planos coronal y sagital oblicua de la rama mandibular y es una opción donde sistemas más sofisticados son poco accesibles ${ }^{51}$. La tomografía computarizada de haz cónico (TCHC) genera una menor dosis de radiación que la TC y permite visualizar la ATM en múltiples cortes sagitales, coronales y axiales de manera que pueden evaluarse todas sus superficies (Figura 3), además, es capaz de reconstruir imágenes tridimensionales a través de softwares permitiendo disponer de una visualización más completa ${ }^{2,12}$ (Figura 4).

La TCHC permite observar los tejidos duros que componen a la ATM, pero tiene como limitante no reflejar a los tejidos blandos como el disco articular y músculos. Comert y cols. identificaron por medio de TCHC que los hallazgos más frecuentes en 117 ATM de pacientes diagnosticados con OA fueron: aplanamiento condilar $(92,3 \%)$, erosión $(94 \%)$, osteofitos condilares $(79,5 \%)^{20}$; estos datos son similares a los reportados por Martínez y cols. ${ }^{9}$; sin embargo, difieren de los reportes de Bechtold y cols. quienes encontraron que los hallazgos más comunes fueron esclerosis $(30,2 \%)$ y erosión $(29,3 \%)^{52}$. Wiberg y cols. sugieren que los distintos signos encontrados en la OA de la ATM pueden representar estadios distintos de la enfermedad, siendo que las lesiones erosivas pueden indicar cambios tempranos, mientras que la presencia de aplanamiento y osteofitos indican cambios tardíos; por otro lado, la presencia de esclerosis refleja un estadio de reparación ósea ${ }^{53}$.

La resonancia magnética (RM) permite la visualización de los tejidos duros y blandos que componen la ATM, especialmente el disco $\operatorname{articular}^{1,2}$. Ikeda señala que el uso combinado de TCHC y RM es sinérgico, permitiendo observar cambios en la posición condilar dentro 


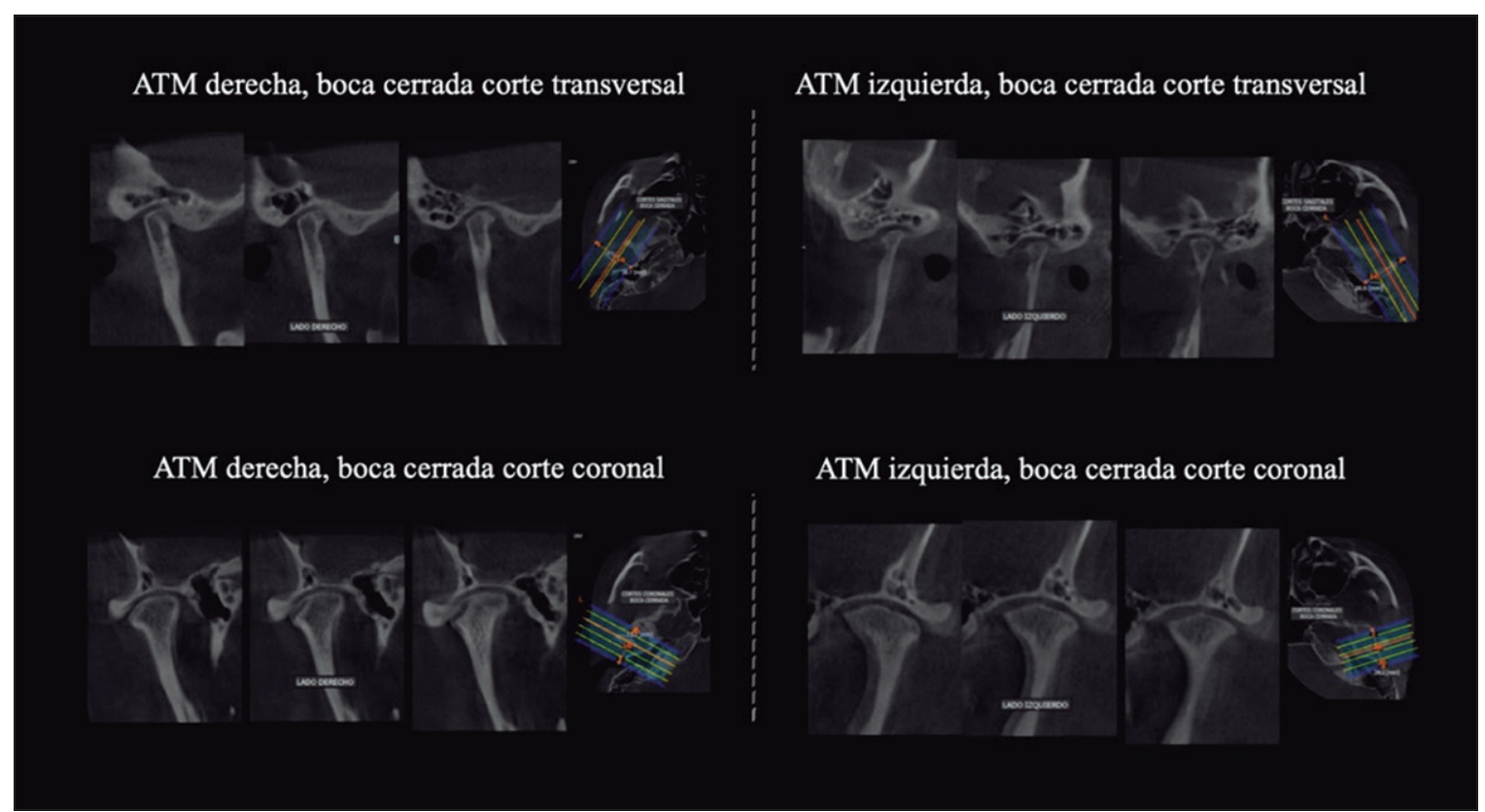

Figura 3. Estudio tomográfico de haz cónico, donde se observan cambios morfológicos compatibles con osteoartritis de la articulación temporomandibular (ATM). En cortes coronal y sagital de ATM derecha en boca cerrada se observa facetamiento y engrosamiento de las corticales óseas articulares y disminución de espacio articular anterior. En ATM izquierda en cortes coronal y sagital a boca cerrada se observa aplanamiento moderado de las superficies articulares y erosión del polo anterior del cóndilo.

Figura 4. Reconstrucción 3D de estudio tomográfico de haz cónico, donde se observa articulación temporomandibular (ATM) izquierda con aplanamiento de superficie condilar, compatible con osteoartritis de la ATM.

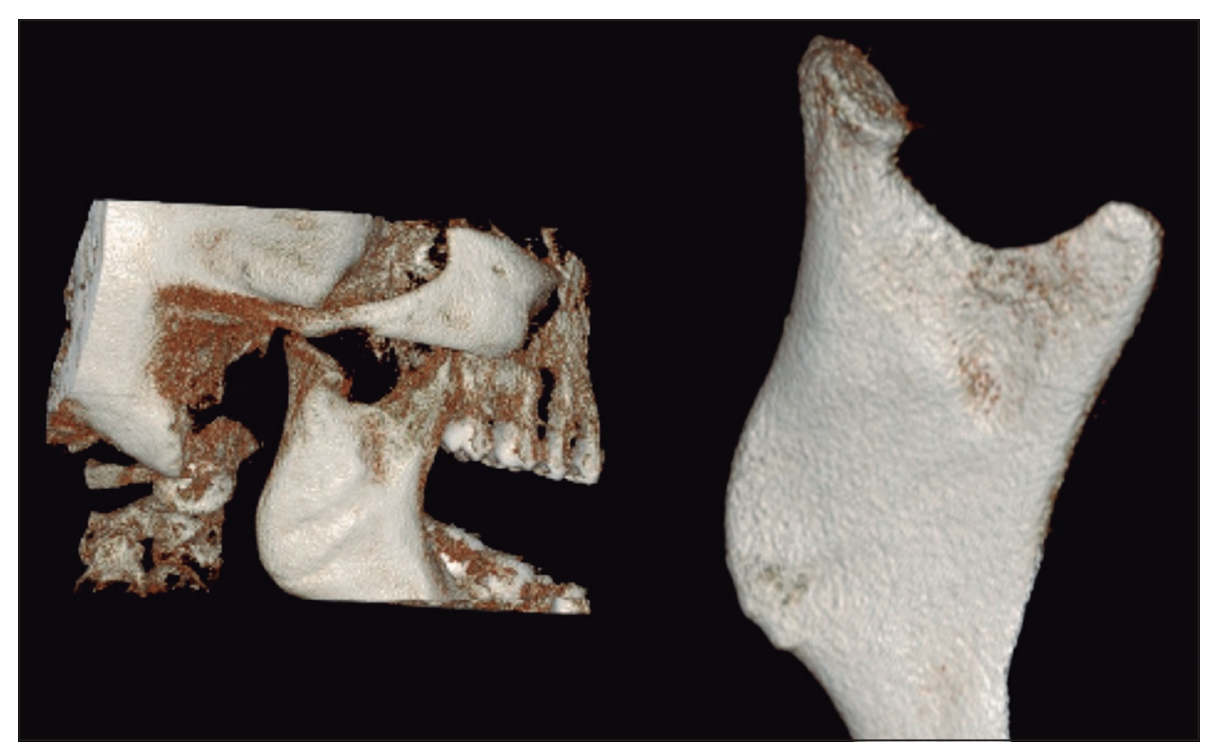

de la fosa y cómo estos cambios se relacionan a las variaciones morfológicas en los tejidos duros y blandos de la estructura articular ${ }^{12}$. Particularmente la RM es la herramienta diagnóstica ideal para identificar la dirección y extensión del desplazamiento de disco (DD) (Figura 5).

Ishida y cols. identificaron que en situaciones en donde se presentaban desplazamientos persistentes de disco sin reducción, era común 


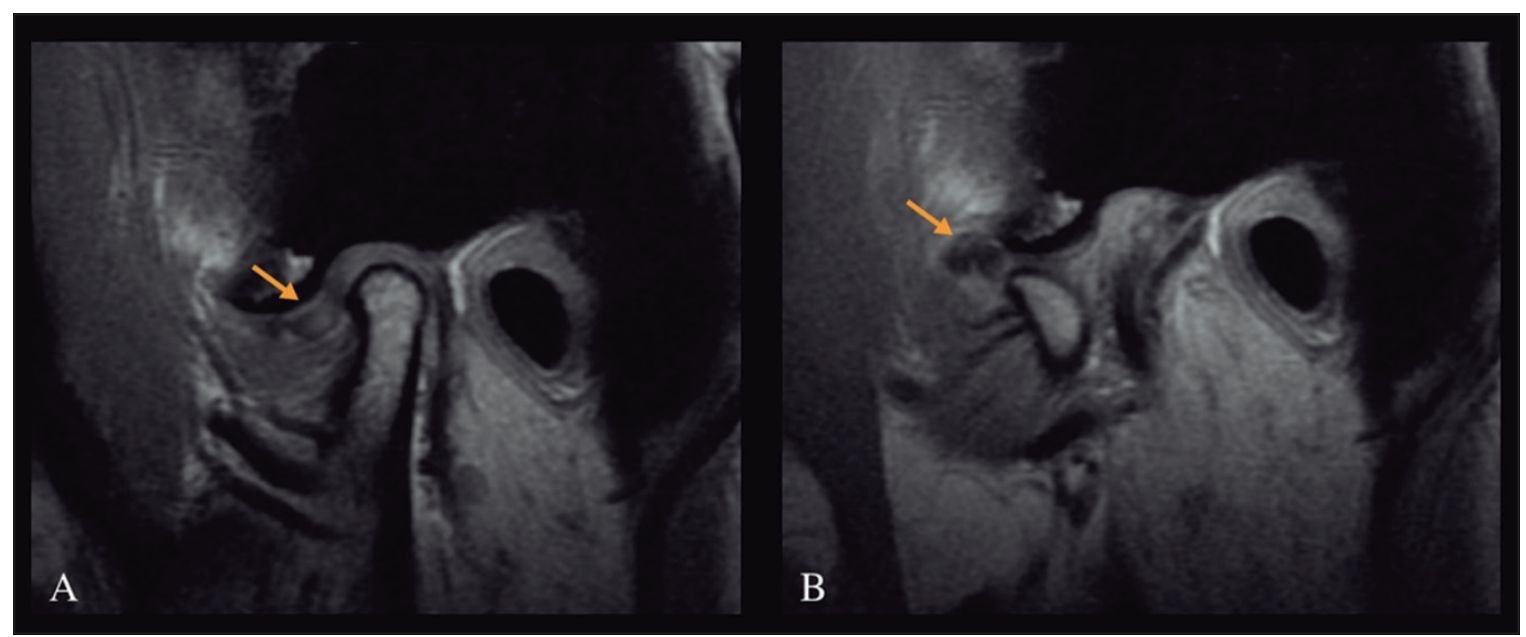

Figura 5. Imagen de resonancia magnética de la articulación temporomandibular (ATM). A: ATM derecha en boca cerrada donde se observa desplazamiento anterior completo de disco articular. B: ATM derecha en boca abierta donde se observa desplazamiento anterior completo sin reducción de disco articular

observar aplanamiento y deformación de la eminencia articular, inclusive en aquellos casos donde los signos y síntomas de disfunción de la ATM habían disminuido ${ }^{54}$. Esto sugiere la importancia del empleo de RM en el diagnóstico de la OA de la ATM, ya que la OA es comúnmente observada en articulaciones con DD sin reducción ${ }^{55}$. Otra característica que puede ser observada por medio de la RM es la efusión articular, que se define como una colección patológica de fluido articular en los espacios articulares ${ }^{48}$, se distingue como un incremento en la señal sobre T2 en las imágenes de RM y es más común en las articulaciones que presentan $\mathrm{DD}$ sin reducción ${ }^{56}$. Las imágenes de RM de la ATM deben ser obtenidas en los planos sagital y coronal oblicuo, que permiten una correcta captura de las relaciones espaciales entre el disco, el cóndilo y la fosa. El protocolo de RM ponderada en T1 es el más empleado para las posiciones a boca cerrada y boca abierta permitiendo observar la morfología de los componentes de la ATM, mientras que la ponderación en T2 permite la detección de efusión o edema de la médula ósea; la indicación de contraste debe ser reservada cuando se sospeche de malignidad a nivel de los tejidos de la $\mathrm{ATM}^{57}$.

En resumen, esta patología se presenta con mayor frecuencia en mujeres entre los 40 a 75 años de edad, que presentan alguna parafunción como el bruxismo, oclusión inestable, desarreglos internos de la articulación o predisposición hereditaria a sufrir artrosis; además la OA se caracteriza por la presencia de ruidos articulares y limitación de los movimientos mandibulares, mientras que la presencia de dolor es común solo en estadios iniciales; el estudio por imágenes evidencia una erosión a nivel de la superficie articular con subsecuente remodelación y rarefacción, pudiendo evidenciarse aplanamiento condilar y formación de osteofitos.

\section{Tratamiento}

Una vez que se ha establecido el diagnóstico de OA de la ATM, la elección del tratamiento responderá al estadio de la enfermedad, los signos y síntomas clínicos y los factores de riesgo preexistentes. El tratamiento debe estar enfocado a suprimir el proceso inflamatorio activo, preservar la función, prevenir el incremento de la desarmonía estructural y el alivio del dolor ${ }^{58}$.

\section{Tratamiento no invasivo}

Las férulas de estabilización (FE) articular han sido empleadas con frecuencia para el tratamiento de los DTM ${ }^{1}$. El uso de estas férulas redistribuye las fuerzas, relaja los músculos elevadores, reduce el bruxismo y disminuye 
la carga articular y, por lo tanto, son útiles para disminuir el dolor ${ }^{12}$. Dentro de estos dispositivos existen aquellos denominados como férulas de reposicionamiento anterior ${ }^{2}$, diseñados con el propósito de recapturar el disco en los casos de desplazamiento anterior de disco sin reducción (Figura 6).

Ok y cols. evaluaron a través de super-imposiciones de TCHC los cambios a nivel óseo de la fosa glenoidea relacionados al uso de FE, encontrando que los pacientes que emplearon FE tuvieron mejoras en la integridad del hueso cortical y experimentaron una disminución de cargas excesivas en la $\mathrm{ATM}^{59}$. En otro estudio Ok y cols. demostraron que el uso de FE induce una remodelación ósea favorable con mejora en el grosor de la cortical en la sección anteromedial, anterointermedia y anterolateral del cóndilo en pacientes con OA de la $\mathrm{ATM}^{60}$. Estos resultados sugieren que las FE son una buena alternativa en pacientes con OA de la ATM y su uso se recomendaría como primer abordaje terapéutico no invasivo.

La mayor producción de citoquinas pro-inflamatorias en la fisiopatología de la OA de la ATM conllevaría al uso de AINEs por sus efectos de la inhibición de la ciclooxigenasa. AINEs como el ibuprofeno, naproxeno y diclofenaco pueden ser empleados por sus buenas propiedades farmacocinéticas y poseer propiedades analgésicas y antiinflamatorias; se recomienda que los pacientes las tomen de manera regular y no solo en caso de dolor, manteniendo niveles séricos del compuesto durante 7 a 10 días, pudiendo disminuir la dosis luego de este periodo de tiempo para disminuir los efectos adversos a nivel del tracto gastrointestinal ${ }^{61}$. Se ha descrito que el uso de los inhibidores de la COX-2 en los desórdenes degenerativos de la ATM tienen un efecto protector manteniendo la homeostasis de los condrocitos de los cóndilos mandibulares ${ }^{58}$. El mecanismo de acción de estos fármacos está relacionado con la inhibición selectiva del ácido araquidónico a prostaglandina $\mathrm{H} 2$ por $\mathrm{COX}-2$ sin disminuir los efectos protectores de la COX-1 a nivel de la mucosa gástrica ${ }^{62}$. Celecoxib es uno de estos inhibidores de la COX-2 frecuentemente empleado para la reducción del dolor en pacientes con desórdenes degenerativos de la ATM, recomendándose su uso dos veces por día en una dosis de $200 \mathrm{mg}^{61}$; Ta y Dionne realizaron un ensayo aleatorizado para comparar la efectividad de celecoxib versus naproxeno para el tratamiento del dolor en la ATM, demostrando que luego de 3 semanas de tratamiento celexocib mostró una mejor reducción del dolor ${ }^{63}$. En estos casos también se puede indicar el uso de meloxicam con una dosis ajustada a 7,5-15 mg por día por 2 semanas, pudiendo extenderse su uso a 4 semanas $^{61}$.

Otra alternativa de tratamiento es la pres-
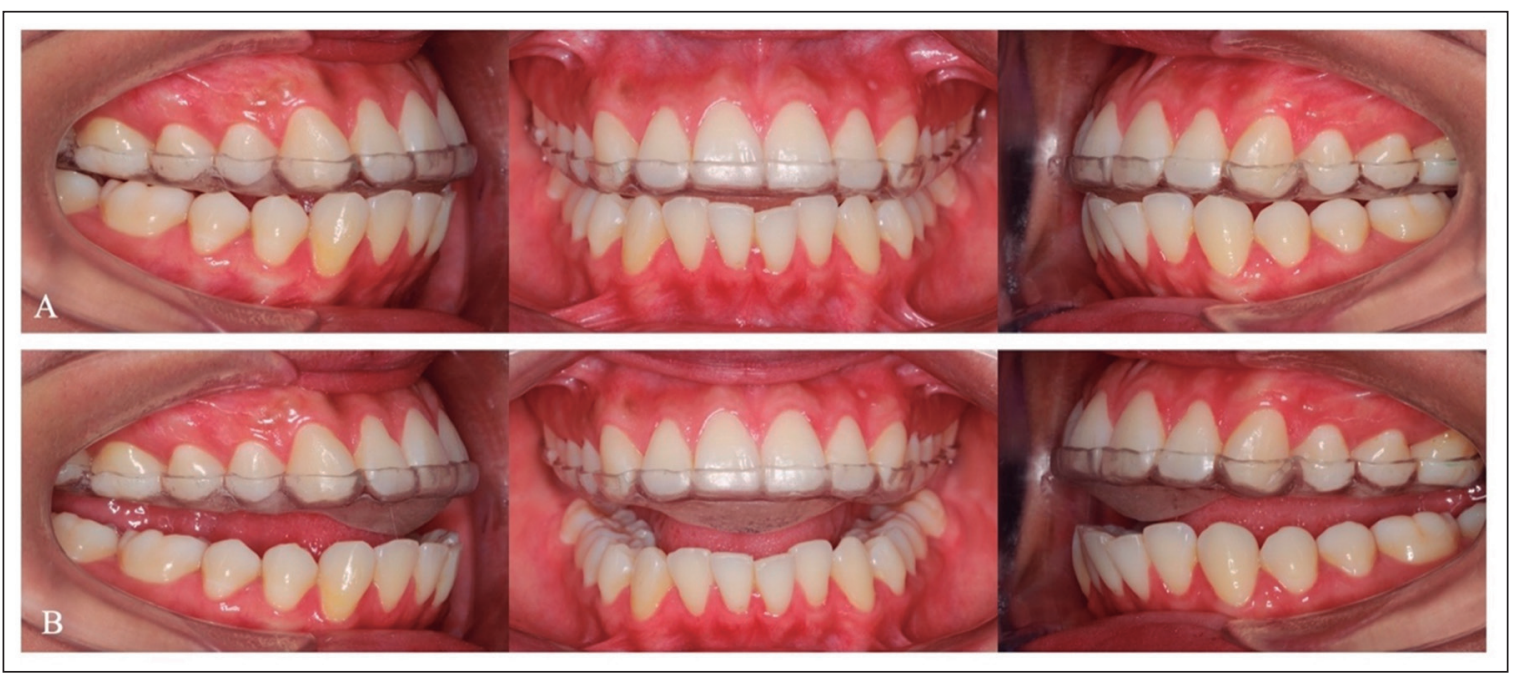

Figura 6. A: Paciente con desplazamiento anterior de disco sin reducción usando férula de reposicionamiento mandibular en boca cerrada. B: Mismo paciente a boca abierta, donde se observa el detalle de la férula. 
cripción de corticoesteroides administrados por vía oral en combinación con AINEs inhibidores selectivos de COX-2. Esta combinación ha demostrado la disminución de los efectos adversos a nivel gastrointestinal y el incremento del efecto antiinflamatorio ${ }^{2}$. La inyección directa de corticoides diluida con lidocaína en la ATM permite una reducción del dolor e incremento de la función entre 4 a 6 semanas ${ }^{64}$.

Dentro de los medicamentos modificadores de la estructura se ha reportado al sulfato de condroitina y al sulfato de glicosamina ${ }^{65}$, el mecanismo de acción de estos medicamentos para el tratamiento de la OA de la ATM no ha sido elucidado por completo; sin embargo, se sabe que estos medicamentos estimulan la síntesis de glicosaminoglicanos en condrocitos, y la acción antiproteasa del sulfato de condroitina brinda mayor eficacia en el retardo del proceso degenerativo ${ }^{66,67}$. Por otro lado, algunos estudios no han mostrado efectividad en el empleo de estos medicamentos para la mejora de los procesos degenerativos de la $\mathrm{ATM}^{65,68}$.

Otra estrategia terapéutica es el uso de fisioterapia con agentes por calor húmedo para la reducción de dolor y rigidez o aplicación de compresas frías por periodos de 15 a 20 minutos ${ }^{2}$, que permiten y facilitan el ejercicio terapéutico a través de movimientos mandibulares controlados y técnicas de relajación; estos ejercicios incluyen la abertura de la boca, laterotrusión y protrusión mandibular bajo resistencia, lo que mejora la movilidad articular, la circulación sanguínea local y previene la formación de adhesiones ${ }^{69}$, esta terapia puede ser realizada por el kinesiólogo u odontólogo especialista en disfunción craneomandibular ${ }^{1,2}$. También se han propuestos otros métodos como el ultrasonido y la estimulación electrogalvánica para la disminución inflamatoria de la región afectada ${ }^{10}$. Ramakrishnan y cols. evaluaron la eficacia de la aplicación de fonoforesis con gel de aceclofenaco y de ultrasonido en pacientes con desórdenes temporomandibulares, sus resultados mostraron que ambos métodos fueron efectivos en la reducción del dolor luego de la aplicación de tres veces por dos semanas sin mostrar diferencias significativas ${ }^{70}$.

\section{Tratamiento mínimamente invasivo}

La aplicación de ácido hialurónico intracapsular disminuye la degeneración articular, además es empleado como un suplemento de viscosidad que normaliza la actividad bioquímica en la articulación al sustituir las moléculas de hialuronato de bajo peso molecular por las de alto peso molecular. El ácido hialurónico en inyección intraarticular es propuesta para disminuir la fricción como lubricante de la articulación; Manfredini y cols. evaluaron la aplicación de inyecciones de ácido hialurónico asociado a artrocentesis en 76 pacientes con OA de la ATM, sus resultados indican que la terapia combinada muestra mejoras en la sintomatología de los pacientes que se mantuvo en un período de seguimiento de hasta 6 meses $^{71}$. Sin embargo, aún no hay estudios concluyentes al respecto, así como tampoco relacionados al efecto en el retraso del daño de la articulación ${ }^{58}$. En una revisión sistemática se concluyó que no hay diferencia en la efectividad entre uso de inyecciones intraarticulares que consisten en hialuronato de sodio o preparaciones de corticoesteroides y una reducción equivalente del dolor con diclofenaco sódico en comparación con las férulas oclusales. La glucosamina parece ser tan efectiva como el ibuprofeno para el tratamiento de la OA de la ATM $^{72}$.

Recientemente Ok y cols. evaluaron la eficacia de inyecciones intraarticulares de hormona de crecimiento en OA de ATM de ratas inducidas con iodoacetato monosódico, observando el incremento en niveles de factor de crecimiento insulínico de tipo 1 (IGF-1) y hormona de crecimiento a nivel articular lo que redujo la ocurrencia de OA sin afectar el crecimiento del hueso condilar, estos resultados sugieren el potencial uso de la hormona de crecimiento para el tratamiento de la OA de $\mathrm{ATM}^{73}$. En consecuencia, existen diversas estrategias terapéuticas para el manejo conservador de la OA de la ATM que incluyen el uso de dispositivos oclusales para disminuir la carga articular y el empleo de AINEs y corticoesteroides administrados por vía oral, además del uso de inyecciones intraarticulares de corticoides y ácido hialurónico para el control del dolor y mejora de la movilidad.

\section{Tratamiento invasivo}

Las intervenciones quirúrgicas solo deben ser consideradas cuando todos los tratamientos conservadores fracasaron. Las intervenciones quirúrgicas incluyen procedi- 
mientos como la artroscopia, condilectomía, artroplastia, reemplazo autógeno de disco o reemplazo total de la articulación también han sido propuestas para el manejo de la OA de la $\mathrm{ATM}^{73-76}$. La artroscopia permite la realización de sinovectomía y disectomía, que se emplea como tratamiento preventivo cuando el dolor y la deficiencia funcional persisten en la ATM evitando el deterioro del cartílago y el hueso articular; la membrana sinovial se reconstituye entre 60 a 90 días sin presentar sintomatología con una tasa de éxito del 73\% en los casos tratados ${ }^{77}$. Abordajes abiertos como la artroplastia permiten la remodelación de las superficies articulares para eliminar los defectos óseos encontrados en la OA de la $\mathrm{ATM}^{10}$. Recientemente el uso de membrana amniótica humana ha sido propuesto como material de interposición en procedimientos de artroplastia de la ATM con OA, demostrando una resolución del dolor y mejora considerable en la movilidad articular y remodelación de la fosa glenoidea con formación de hueso, estos resultados sugieren la posibilidad de inducir la formación de hueso ectópico en esta región con el uso de este abordaje ${ }^{78}$. La elección e indicación de estos procedimientos deben cumplir algunos criterios como la compatibilidad biológica, resistencia mecánica y la biomecánica articular ${ }^{6,69}$.

\section{Conclusión}

La osteoartritis de la ATM es un proceso degenerativo bastante frecuente en los pacientes con requerimiento de tratamiento odontológico, pero a la vez subdiagnosticado. Los factores etiológicos asociados a los cambios degenerativos producidos en la ATM incluyen los procesos de enfermedad sistémica, factores hormonales y factores relacionados con el incremento de la carga mecánica sobre la articulación. Un entendimiento apropiado del comportamiento biomecánico de la articulación es importante para poder diagnosticarla y definir las estrategias terapéuticas para su manejo. Las metas de tratamiento para los pacientes con OA de la ATM incluyen la restitución de la función y la reducción del dolor y las distintas modalidades pueden ir desde terapia no invasiva, mínimamente invasiva, hasta la cirugía de la ATM.

\section{Bibliografía}

1. Okeson J. Tratamiento de oclusión y afecciones temporomandibulares. $7^{\text {a }}$ Edición. España: Elsevier; 2013.

2. Wrigth E. Manual de trastornos temporomandibulares. $3^{\text {ra }}$ Edición. Caracas: AMOLCA; 2016.

3. Ranco C, Waldron T. TMJ osteoarthritis: a new approach to diagnosis. Am J Phys Anthropol. 2012;148(1):45-53. doi: 10.1002/ajpa.22039.

4. Poole AR. Osteoarthritis as a whole joint disease. HSSJ. 2012;8(1): 4-6. doi: 10.1007/s11420-011-92486.

5. Sinusas K. Osteoarthritis: diagnosis and treatment. Am Fam Physician. 2012;85(1):49-56.

6. Liu F, Steinkeler A. Epidemiology, diagnosis, and treatment of temporomandibular disorders. Dent Clin North Am. 2013;57(3):465-79. doi: 10.1016/j. cden.2013.04.006.

7. Dimitroulis G. The prevalence of osteoarthrosis in cases of advanced internal derangement of the temporomandibular joint: a clinical, surgical and histological study. Int J Oral Maxillofac Surg. 2005;34(4):345-9. doi: 10.1016/j.ijom.2004.10.013.

8. Dijkgraaf LC, de Bont LG, Boering G, Liem RS. The structure, biochemistry, and metabolism of osteoarthritic cartilage: a review of the literature. J Oral maxillofac Surg. 1995;53(10):1182-92. doi: 10.1016/0278-2391(95)90632-0.

9. Martínez Blanco M, Bagán JV, Fons A, Poveda Roda R. Osteoarthrosis of the temporomandibular joint. A clinical and radiological study of 16 patients. Med Oral. 2004;9(2):110-15,106-10.

10. Tanaka E, Detamore MS, Mercuri LG. Degenerative disorders of the temporomandibular joint: etiology diagnosis, and treatment. J Dent Res. 2008;87(4):296307. doi: 10.1177/154405910808700406.

11. Tanaka E, Kikuchi K, Sasaki A, Tanne K. An adult case of TMJ osteoarthrosis treated with splint therapy and the subsequent orthodontic occlusal reconstruction: Adaptive change of the condyle during the treatment. Am J Orthod Dentofacial Orthop. 2000;118(5):566-71. doi: 10.1067/ mod.2000.93966.

12. Ikeda K. TMJ 1st Orthodontics, concepts, mechanics, and stability. First. Tokyo: Topnoch Kikuda; 2014.

13. Bansal M. Prevalence and diagnostic features of osteoarthrosis of the temporomandibular joint: a review. Int J Res Orthop. 2016;2(1):1-4.

14. Bernhardt O, Biffar R, Kocher T, Meyer G. Prevalence and clinical signs of degenerative temporomandibular joint changes validated by magnetic resonance imaging in a non-patient group. Ann Ant. 2007;189(4):342-6. doi: 10.1259/dmfr/16270943.

15. Schmitter M, Essig M, Seneadza V, Balke Z, Schröder J, Rammelsberg P. Prevalence of clinical 
and radiographic signs of osteoarthrosis of the temporomandibular joint in an older person community. Dentomaxillofac Radiol. 2010;39(4):2314. doi: $10.1259 / \mathrm{dmfr} / 16270943$.

16. Das SK. TMJ osteoarthritis and early diagnosis. J Oral Biol Craniofac Res. 2013;3(3):109-110. doi: 10.1016/j. jobcr.2013.10.003.

17. Torrealba TM, Normandin UP, Gúzman ZC, Kuramochi DG. Prevalencia y distribución de signos degenerativos en cóndilo de la ATM presentes en radiografías panorámicas en población chilena. Int $J$ Mophol. 2018;36(4):1519-1524.

18. Lee DG, Kim TW, Kang SC, Kim ST. Estrogen receptor gene polymorphism and craniofacial morphology in female TMJ osteoarthritis patients. Int J Oral Maxillofac Surg. 2006;35(2):165-9. doi: 10.1016/j.ijom.2005.06.009.

19. Kang SC, Lee DG, Choi JH, Kim ST, Kim YK, Ahn HJ. Association between estrogen receptor polymorphism and pain susceptibility in female temporomandibular joint osteoarthritis patients. Int J Oral Maxillofac Surg. 2007;36(5):391-4. doi: 10.1016/j.ijom.2006.12.004.

20. Cömert Kiliç S, Kiliç N, Sümbüllü MA. Temporomandibular joint osteoarthritis: Cone beam computed tomography findings, clinical features, and correlations. Int J Oral Maxillofac Surg. 2015;44(10):1268-74. doi: 10.1016/j.ijom.2015.06.023.

21. LeResche L. Epidemiology of temporomandibular disorders: implications for the investigation of etiologic factors. Crit Rev Oral Biol Med. 1997;8(3):291-305. doi: 10.1177/10454411970080030401.

22. Ong TK, Franklin CD. A clinical and histopathological study of osteoarthrosis of temporomandibular joint. Br J Oral maxillofac Surg. 1996; 34(2):186-92. doi: 10.1016/s02664356(96)90375-7.

23. Bessa-Nogueira RV, Vasconcelos BC, Duarte AP, Góes PS, Bezerra TP. Targeted assessment of the temporomandibular joint in patients with rheumatoid arthritis. J Oral Maxillofac Surg. 2008;66:1804-11. doi: 10.1016/j.joms.2007.08.037.

24. Stoll ML, Kau CH, Waite PD, Cron RQ. Temporomandibular joint arthritis in juvenile idiopathic arthritis, now what? Pediatr Rheumatol Online J. 2018;16(1):32.

25. Goupille P, Fouquet B, Goga D, Cotty P, Valat JP. The temporomandibular joint in rheumatoid arthritis: correlations between clinical and tomographic features. J Dent. 1993;21(3):141-6. doi: 10.1186/ s12969-018-0244-y.

26. Ueno T, Kagawa T, Kanou M, et al. Pathology of the temporomandibular joint of patients with rheumatoid arthritis-case report of secondary amyloidosis and macrophage populations. J Craniomaxillofac Surg. 2003;31(4):252-6. doi: 10.1016/s1010-5182(03)00031-3.

27. Keersmaekers K, De Boever JA, Van Den Berghe L. Otalgia in patients with temporomandibular joint disorders. J Prosthet Dent. 1996;75(1):72-76. doi: 10.1016/s0022-3913(96)90421-7.

28. Manfredini D, Olivo M, Ferronato G, Marchese R, Martini A, Guarda-Nardini L. Prevalence of tinnitus in patients with different temporomandibular disorders symptoms. Int Tinnitus J. 2015;19(2):47-51. doi: 10.5935/0946-5448.20150008.

29. Macedo J, Doi M, Oltramari-Navarro PV, et al. Association between Ear Fullness, Earache, and Temporomandibular Joint Disorders in the Elderly. Int Arch Otorhinolaryngol. 2014;18(4):383-6. doi: 10.1055/s-0034-1385844.

30. Molina OF, Aquilino RN, Rank R, Santos ZC, Eid NLM, Tavares PG. Is inflammation a mechanism in arthrogenic TMJ Otalgia? Rev Neuroscience. 2011;19(4):632-641.

31. Ikeda K, Kawamura A. Disc displacement and changes in condylar position. Dentomaxillofac Radiol. 2013; 42(3): 11-7. doi: 10.1259/dmfr/84227642.

32. Dias IM, Cordeiro PC, Devito KL, Tavares ML, Leite IC, Tesch R de S. Evaluation of temporomandibular joint disc displacement as a risk factor for osteoarthrosis. Int J Oral Maxillofac Surg. 2016; 45(3): 313-7. doi: 10.1016/j.ijom.2015.09.016.

33. Arnett GW, Gunson MJ. Risk factors in the initiation of condylar resorption. Semin Orthod. 2013;19(2): 81-88.

34. Arnett GW, Milam SB, Gottesman L. Progressive mandibular retrusion-idiopathic condylar resorption. Part I. Am J Orthod Dentofacial Orthop. 1996; 110(1): 8-15. doi: 10.1016/s0889-5406(96)70081-1.

35. Milam SB. Pathogenesis of degenerative temporomandibular joint arthritis. Odontology. 2005; 93(1): 7-15. doi: 10.1007/s10266-005-0056-7.

36. Lawrence RC, Helmick CG, Arnett FC, et al. Estimates of the prevalence of arthritis and selected musculoskeletal disorder in the United States. Arthritis Rheum. 1998;41(5):778-799.

37. Takano Y, Moriwake Y, Tohno Y, et al. Age-related changes of elements in the human articular disk of the temporomandibular joint. Biol Trace Elem Res. 1999;67(3):269-76. doi: 10.1007/BF02784426.

38. Berger M, Szalewski L, Bakalczuk M, Bakalczuk G, Bakalczuk S, Szutnik J. Association between estrogen levels and temporomandibular disorders: a systematic literature review. Prz Menopauzalny. 2015;14(4):26070. doi: 10.5114/pm.2015.56538.

39. Guarda-Nardini L, Piccotti F, Mogno G, Favero L, Mandredini D. Age-related differences in temporomandibular disorder diagnoses. Cranio. 2012;30(2):103-9. doi: 10.1179/crn.2012.015.

40. Dumbuya A, Gomes AF, Marchini L, Zeng E, Comnick CL, Melo SLS. Bone changes in the temporomandibular joints of older adults: A cone- 
beam computed tomography study. Spec Care Dentist. 2020;40(1):88-89.

41. Mobasheri A, Batt M. An update on the pathophysiology of osteoarthritis. Ann Phys Rehabil Med. 2016;59(5-6):333-339. doi: 10.1016/j. rehab.2016.07.004.

42. Sánchez NJC, López ZDF. Fisiopatología celular de la osteoartritis: el condrocito articular como protagonista. Iatreia. 2011;24(2):167-178.

43. Glyn-Jones S, Palmer AJ, Agricola R, et al. Osteoarthritis. Lancet. 2015;386(9991):376-87. doi: 10.1016/S0140-6736(14)60802-3.

44. Sellam J, Berenbaum F. The role of synovitis in pathophysiology and clinical symptoms of osteoarthritis. Nat Rev Rheumatol. 2010;6(11):625-35. doi: 10.1038/nrrheum.2010.159.

45. Cevidanes LH, Walker D, Schilling J, et al. 3D osteoarthritic changes in TMJ condylar morphology correlates with specific systemic and local biomarkers of disease. Osteoarthritis Cartilage. 2014;22(10):165767. doi: 10.1016/j.joca.2014.06.014.

46. Vernal R, Velásquez E, Gamonal J, García-Sanz JA, Silva A, Sanz M. Expression of proinflammatory cytokines in osteoarthritis of the temporomandibular joint. Arch Oral Biol. 2008;53(10):910-5. doi: 10.1016/j.archoralbio.2008.04.004.

47. López-Armada MJ, Carames B, Cillero-Pastor B, Blanco García FJ. Fisiopatología de la arthrosis ¿Cuál es la actualidad? Rev Esp Reumatol. 2004;31(6):37993.

48. Wang XD, Zhang JN, Gan YH, Zhou YH. Current understanding of pathogenesis and treatment of TMJ osteoarthritis. J Dent Res. 2015;94(5):666-73. doi: 10.1177/0022034515574770.

49. Rahmati M, Mobasheri A, Mozafari M. Inflammatory mediators in osteoarthritis: A critical review of the state-of-the-art, current prospects, and future challenges. Bone. 2016;85:81-90. doi: 10.1016/j. bone.2016.01.019.

50. Emshoff R, Innerhofer K, Rudisch A, Bertram S. The biological concept of "internal derangement and osteoarthrosis": a diagnostic approach in patients with temporomandibular joint pain? Oral Surg Oral Med Pathol Oral Radiol Edod. 2002;93(1):39-44.

51. Boeddinghaus R, Whyte A. Computed tomography of the temporomandibular joint. J Med Imaging Radiat Oncol. 2013;57(4):448-54. doi: 10.1111/17549485.12021.

52. Bechtold TE, Saunders C, Decker RS, et al. Osteophyte formation and matrix mineralization in a TMJ osteoarthritis mouse model are associated with ectopic hedgehog signaling. Matrix Biol. 2016;5254:339-354. doi: 10.1016/j.matbio.2016.03.001.

53. Wiberg B, Wanman A. Signs of osteoarthrosis of the temporomandibular joints in young patients: a clinical and radiographic study. Oral Surg Oral Med Oral Pathol Oral Radiol Endod. 1998;86(2):158-62. doi: 10.1016/s1079-2104(98)90118-4.

54. Ishida Y, Kurita K, Ogi N, et al. Primary treatment for osteoarthritis of the temporomandibular joint by combination therapy of arthrocentesis, mouth opening exercises, and a non-steroidal antiinflammatory drug regimen. Asian J Oral Maxillofac Surg. 2003;15(1):14-8.

55. Sano T, Yajima A, Otonari-Yamamoto M, Wakoh M, Katakura A. Interpretation of images and discrepancy between osteoarthritic findings and symptomatology in temporomandibular joint. Jpn Dent Sci Rev. 2008;44(1):83-9.

56. Yamamoto M, Sano T, Okano T. Magnetic resonance evidence of joint fluid with temporomandibular joint disorders. J Comput Assist Tomogr. 2003;27(5):694-8. doi: 10.1097/00004728-200309000-00006.

57 Tamimi D, Jalali E, Hatcher D. Temporomandibular joint imaging. Radiol Clin N Am. 2018;56(1):157-175. doi: 10.1016/j.rcl.2017.08.011.

58 Kalladka M, Quek S, Heir G, Eliav E, Mupparapu $\mathrm{M}$, Viswanath A. Temporomandibular joint osteoarthritis: diagnosis and long-term conservative management: a topic review. J Indian Prosthodont Soc. 2014;14(1):6-15. doi: 10.1007/s13191-013-0321-3.

59 Ok SM, Jeong SH, Ahn YW, Kim Y Il. Effect of stabilization splint therapy on glenoid fossa remodeling in temporomandibular joint osteoarthritis. J Prosthodont Res. 2016;60(4):301-7. doi: 10.1016/j.jpor.2016.03.001.

60. Ok SM, Lee J, Kim Y IL, Lee JY, Kim KB, Jeong $\mathrm{SH}$. Anterior condylar remodeling observed in stabilization splint therapy for temporomandibular joint osteoarthritis. Oral Surg Oral Med Oral Pathol Oral Radiol. 2014;118(3):363-70. doi: 10.1016/j. oooo.2014.05.022.

61. Ouanounou A, Golberg M, Haas DA. Pharmacotherapy in temporomandibular disorders: A Review. J Can Dent Assoc. 2017;83:h7.

62. López-Zaragoza JL, Gulias-Herrero A, GutiérrezCirios C, et al. Actitud ante los inhibidores selectivos de COX-2. Rev Invest Clín. 2005;57(1):6-12.

63. Ta LE, Dionne RA. Treatment of painful temporomandibular joints with a cyclooxygenase-2 inhibitor: a randomized placebo-controlled comparison of celecoxib to naproxen. Pain. 2004;111(1-2):13-21. doi:10.1016/j.pain.2004.04.029.

64. Fredriksson L, Altergren P, Kopp S. Serotonergic mechanisms influence the response to glucocorticoid treatment in TMJ arthritis. Mediators Inflamm. 2005;2005(4):194-201. doi: 10.1155/MI.2005.194.

65. Machado E, Machado P, Cunali PA. Utilização de sulfato de condroitina e sulfato de glicosamina nas alterações degenerativas da ATM: uma revisão sistematica. Dental Press J. Orthod. 2012;17(4):1-5.

66. Nguyen P, Mohamed SE, Gardiner D, Salinas T. A randomized double-blind clinical trial of the effect of chondroitin sulfate and glucosamine 
hydrochloride on temporomandibular joint disorders: a pilot study. Cranio. 2001;19(2):130-9. doi: 10.1080/08869634.2001.11746162.

67. Haghighat A, Behnia A, Kaviani N, Khorami B. Evaluation of glucosamine sulfate and ibuprofen effects in patients with temporomandibular joint osteoarthritis symptom. J Res Pharm Pract. 2013;2(1):34-39. doi: 10.4103/2279-042X.114087.

68. Cahlin BJ, Dahlstrom L. No effect of glucosamine sulfate on osteoarthritis in the temporomandibular joints - a randomized, controlled, short-term study. Oral Surg Oral Med Oral Pathol Oral Radiol Endod. 2011;112(6):760-6. doi: 10.1016/j.tripleo.2011.06.012.

69. Mercuri GL. Management of temporomandibular joint disorders. J Oral Biol Craniofac Res. 2012;2(3):141-142.

70. Ramakrishnan SN, Aswath N. Comparative efficacy of analgesic gel phonoforesis and ultrasound in the treatment of temporomandibular joint disorders. Indian J Dent Res. 2019;30(4):512-515. doi: 10.4103/ ijdr.IJDR_634_17.

71. Mafredini D, Bonnini S, Arboretti R, Guarda-Nardini L. temporomandibular joint osteoarthritis: an open label trial of 76 patients treated with arthrocentesis plus hyaluronic acid injections. Int J Oral Maxillofac Surg. 2009;38(8):827-34. doi: 10.1016/j. ijom.2009.03.715.

72. de Souza RF, Lovato da Silva CH, Nasser M, Fedorowicz Z, Al-Muharraqi MA. Interventions for the management of temporomandibular joint osteoarthritis. Cochrane Database Syst Rev. 2012;18(4):CD007261. doi: 10.1002/14651858. CD007261.

73. Ok SM, Kim JH, Kim JS, et al. Local injection of growth hormone for temporomandibular joint osteoarthritis. Yonsei Med J. 2020;61(4):331-340. doi: 10.3349/ymj.2020.61.4.331.

74. Martel-Pelletier J, Barr AJ, Cicuttini FM, et al. Osteoarthritis. Nat Rev Dis Primers. 2016;2:16072. doi: 10.1038/nrdp.2016.72.

75. Jones RH. Temporomandibular joint reconstruction with total alloplastic joint replacement. Aust Dent J. 2011;56(1):85-91. doi: 10.1111/j.18347819.2010.01288.x.

76. Felstead AM, Revington PJ. Surgical management of temporomandibular joint ankyloses in ankylosing spondylitis. Int J Rheumatol. 2011;2011:854167. doi: 10.1155/2011/854167.

77. Bjørnland T, Larheim TA. Synovectomy and diskectomy of the temporomandibular joint in patients with chronic arthritic disease compared with diskectomies in patients with internal derangement. A 3-year follow-up study. Eu J Oral Sci. 1995;103(1):27. doi: 10.1111/j.1600-0722.1995.tb00002.x.

78. López-Martos R, Martin-Lozano G, OcetePérez RF, González-Pérez LM, Gutiérrez-Pérez JL, Infante-Cossio P. Application of human amniotic menbrane in temporomandibular joint osteoarthritis. J Craniofac Surg. 2020. doi: 10.1097/ SCS.0000000000006424. 